\title{
Intellectual disability-craniofacial dysmorphism-cryptorchidism syndrome
}

INSERM

\section{Source}

INSERM. (1999). Orphanet: an online rare disease and orphan drug data base. Intellectual disability-craniofacial dysmorphism-cryptorchidism syndrome. ORPHA:329224

Intellectual disability-craniofacial dysmorphism-cryptorchidism syndrome is a rare, genetic, syndromic intellectual disability syndrome characterized by mild to moderate intellectual disability, developmental delay (with speech and language development more severely affected) and facial dysmorphism which typically includes full, arched eyebrows, hypertelorism, down-slanting palpebral fissures, long eyelashes, ptosis, low-set, simple ears, bulbous nasal tip, flat philtrum, wide mouth with downturned corners and thin upper lip and diastema of the teeth. Association with infantile hypotonia, seizures, cryptorchidism in males and congenital abnormalities, including cardiac, cerebral or occular defects, may be observed. 